\title{
Signal of Carotid Intraplaque Hemorrhage on MR T1-Weighted Imaging: Association with Acute Cerebral Infarct
}

\author{
(DD. Yang, (D) Y. Liu, (D). Han, (DD. Li, (D)W. Wang, (D). Li, (D) C. Yuan, and (D) X. Zhao
}

\begin{abstract}
BACKGROUND AND PURPOSE: Identifying the mere presence of carotid intraplaque hemorrhage would be insufficient to accurately discriminate the presence of acute cerebral infarct. We aimed to investigate the association between signal intensity ratios of carotid intraplaque hemorrhage on T1-weighted MR imaging and acute cerebral infarct in patients with hemorrhagic carotid plaques using MR vessel wall imaging.
\end{abstract}

MATERIALS AND METHODS: Symptomatic patients with carotid intraplaque hemorrhage were included. The signal intensity ratios of carotid intraplaque hemorrhage against muscle on T1-weighted, TOF, and MPRAGE images were measured. The acute cerebral infarct was determined on the hemisphere ipsilateral to the carotid intraplaque hemorrhage. The association between signal intensity ratios of carotid intraplaque hemorrhage and acute cerebral infarct was analyzed.

RESULTS: Of 109 included patients (mean, $66.8 \pm 9.9$ years of age; 96 men), 40 (36.7\%) had acute cerebral infarct. Patients with acute cerebral infarct had significantly higher signal intensity ratios of carotid intraplaque hemorrhage on T1-weighted images than those without (Median, 1.44; 25-75 Percentiles, 1.14-1.82 versus Median, 1.27; 25-75 Percentiles, 1.06-1.55, $P=.022$ ). Logistic regression analysis revealed that the signal intensity ratio of carotid intraplaque hemorrhage on T1-weighted images was significantly associated with acute cerebral infarct before (OR, 4.08; 95\% Cl, 1.34-12.40; $P=.013)$ and after $(\mathrm{OR}, 3.34 ; 95 \% \mathrm{Cl}, 1.08-10.31 ; P=.036)$ adjustment for clinical confounding factors. However, this association was not significant when further adjusted for occlusion of the carotid artery $(P=.058)$ and volumes of intraplaque hemorrhage and lipid-rich necrotic core $(P=.458)$.

CONCLUSIONS: The signal intensity ratio of carotid intraplaque hemorrhage on T1-weighted images is associated with acute cerebral infarct in symptomatic patients with carotid hemorrhagic plaques. This association is independent of traditional risk factors but not of the size of plaque composition. The possibility of applying $\mathrm{Tl}$ signals of carotid intraplaque hemorrhage to predict subsequent cerebrovascular ischemic events needs to be prospectively verified.

ABBREVIATIONS: $\mathrm{ACI}=$ acute cerebral infarct; IPH = intraplaque hemorrhage; LRNC = lipid-rich necrotic core; SIR $=$ signal intensity ratio

n China, stroke has become the leading cause of death and disability in recent years. ${ }^{1}$ It is well established that carotid atherosclerotic disease is one of the important causes of ischemic stroke. ${ }^{2}$ Previous studies have demonstrated that the presence of

Received October 30, 2019; accepted after revision February 29, 2020.

From the Beijing Institute of Brain Disorders (D.Y., Y.H., D.L.), Laboratory of Brain Disorders, Ministry of Science and Technology, Collaborative Innovation Center for Brain Disorders, Capital Medical University, Beijing, China; Department of Biomedical Engineering (D.Y., Y.H., D.L., R.L., X.Z.), Center for Biomedical Imaging Research, Tsinghua University School of Medicine, Beijing, China; Department of Radiology (Y.L., W.W.), The Affiliated Hospital of Yangzhou University, Yangzhou University, Yangzhou, China; and Department of Radiology (C.Y.), University of Washington, Seattle, Washington.

Drs Dandan Yang and Yang Liu are co-first authors who contributed equally to this study.

This study was supported by grants from the National Natural Science Foundation of China (81771825), Beijing Municipal Science and Technology Commission (D171100003017003), and Ministry of Science and Technology of China (2017YFC1307904). carotid intraplaque hemorrhage (IPH) is one of the features of vulnerable plaques ${ }^{3}$ and is a strong predictor of cerebrovascular ischemic events. ${ }^{4-7}$ Fryer et $\mathrm{al}^{8}$ reported that, in symptomatic plaques, the incidence of a larger amount of hemorrhage (involving $\geq 50 \%$ of the plaque thickness) was much more common and IPH may occur at many stages in the evolution of the plaque. Investigators found that the presence of IPH was also observed in asymptomatic carotid atherosclerotic plaques and plaques with $0 \%$ stenosis. ${ }^{9,10}$ A prospective study by

Please address correspondence to Xihai Zhao, MD, PhD, Center for Biomedical Imaging Research, Department of Biomedical Engineering, Tsinghua University School of Medicine, Haidian Dstrict, Beijing, 100084, China; e-mail: xihaizhao@tsinghua.edu.cn

-- Indicates open access to non-subscribers at www.ajnr.org

Indicates article with supplemental on-line photo.

http://dx.doi.org/10.3174/ajnr.A6498 


\begin{tabular}{|c|c|c|c|c|c|c|}
\hline & \multicolumn{4}{|c|}{ Carotid Artery Imaging } & \multicolumn{2}{|c|}{ Brain Imaging } \\
\hline & TIWI & T2WI & TOF & MPRAGE & $\mathrm{DWI}^{\mathrm{a}}$ & TIWI \\
\hline Sequence & TSE & TSE & FFE & FFE & EPI & FFE \\
\hline $\mathrm{TR}$ (ms) & 800 & 4800 & 20 & 8.8 & 2724 & 308 \\
\hline TE (ms) & 10 & 50 & 4.9 & 5.3 & 86 & 4.6 \\
\hline Flip angle & $90^{\circ}$ & $90^{\circ}$ & $20^{\circ}$ & $15^{\circ}$ & $90^{\circ}$ & $90^{\circ}$ \\
\hline $\mathrm{FOV}\left(\mathrm{cm}^{2}\right)$ & $14 \times 14$ & $14 \times 14$ & $14 \times 14$ & $14 \times 14$ & $23 \times 23$ & $23 \times 23$ \\
\hline Matrix size & $256 \times 256$ & $256 \times 256$ & $256 \times 256$ & $256 \times 256$ & $128 \times 126$ & $400 \times 256$ \\
\hline Slice thickness (mm ${ }^{\mathrm{b}}$ ) & 2 & 2 & 1 & 1 & 5.5 & 5.5 \\
\hline
\end{tabular}

Note:-FFE indicates fast-field echo; TSE, turbo spin echo; EPI, echo planar imaging; TR, repetition time; TE, echo time; FOV, field of view.

${ }^{a}$ DWI: $b=0,1000 \mathrm{~s} / \mathrm{mm}^{2}$.

${ }^{\mathrm{b}}$ Values represent the acquired slice thickness.

Sun et $\mathrm{al}^{11}$ suggested that carotid IPH could persist for years with no clinical symptoms. However, the presence of IPH could accelerate the progression of atherosclerotic plaque and increase the risk of plaque rupture. ${ }^{12}$ Therefore, identifying the mere presence of IPH would be insufficient to precisely predict the presence of cerebrovascular ischemic events.

T1-weighted MR images have commonly been used to detect IPH due to the degradation of hemorrhage into methemoglobin, which leads to T1-shortening ${ }^{13}$ and correspondingly causes high signal intensity on T1-weighted MR images. Previous studies have indicated the importance of the signal intensity ratios (SIRs) of carotid plaques on different MR images, which could be taken into account for factors beyond the presence of IPH. ${ }^{14,15}$ A recent study by Wang et $\mathrm{al}^{16}$ indicated that the SIRs of carotid IPH on heavily T1-weighted images on the symptomatic side were higher than those on the asymptomatic side in symptomatic patients with bilateral carotid IPHs. However, this study targeted only the patients with bilateral carotid IPHs with small sample sizes and measured SIRs of carotid IPH on one T1 sequence of MPRAGE. In addition, the acute cerebral infarct (ACI) lesions in that study on the symptomatic side were not distinct.

The purpose of this study was to investigate the association between the SIRs of carotid IPH on different T1-weighted MR images and the presence of ipsilateral ACI lesions in patients with hemorrhagic carotid plaques using multicontrast MR vessel wall imaging.

\section{MATERIALS AND METHODS \\ Patients}

All patients were enrolled from a cross-sectional, multicenter study of Chinese Atherosclerosis Risk Evaluation (CARE-II), which aimed to assess the prevalence and characteristics of carotid high-risk atherosclerotic plaques in Chinese patients with recent ischemic stroke or transient ischemic attack using multicontrast MR vessel wall imaging. The study protocol of the CARE-II study has been reported previously. ${ }^{17}$ The inclusion criteria are as follows: 1) 18-80 years of age, 2) a recent stroke or transient ischemic attack ( $<2$ weeks after the onset of symptoms), 3) atherosclerotic plaques in at least one carotid artery determined by B-mode sonography, and 4) carotid plaques with IPH confirmed by MR vessel wall imaging. Patients with the following conditions were excluded from this study: 1) cardioembolic or hemorrhagic stroke, 2) a history of radiation therapy to the neck, or 3) contraindications to MR imaging. All patients underwent carotid multicontrast MR vessel wall imaging and clinically routine brain MR imaging. The demographics and clinical characteristics of all patients including age, sex, body mass index, history of smoking, hypertension, diabetes, hyperlipidemia, statin use, antihypertension medication use, coronary heart disease, and lipid levels were acquired from the medical records. The study protocol was approved by the institutional review board of Tsinghua University and local institutions, and a written consent form was obtained from each subject.

\section{Carotid and Brain MR Imaging}

All patients underwent carotid and brain MR imaging on 3T MR scanners (Achieva TX; Philips Healthcare, Best, the Netherlands) with 8-channel phased array carotid coils and head coils. A standardized multisequence protocol was performed for the carotid artery by acquiring TOF, T1-weighted, and MPRAGE sequences. Carotid artery imaging was centered on the carotid artery bifurcation on the symptomatic side. Standard brain MR images were acquired using T1-weighted and DWI sequences. The imaging parameters are detailed in Table 1.

\section{MR Image Analysis}

All the carotid images were reviewed in consensus by 2 experienced radiologists who had $>3$ years of experience in neurovascular imaging, blinded to clinical information using customdesigned software (CASCADE; University of Washington, Seattle, Washington). ${ }^{18}$ All carotid images were registered with the landmark of carotid bifurcation. The lumen and outer wall boundaries were manually traced. The lumen, wall, total vessel areas, and the mean wall thickness on each axial image were measured. Normalized wall index, defined as wall area divided by the total vessel area, was calculated. The presence or absence of calcification, lipid-rich necrotic core (LRNC), IPH, and fibrous cap rupture at each axial location was identified according to the published criteria, which were validated by histology. ${ }^{19}$ In particular, the identification of carotid IPH was mainly based on a pattern of hyperintense signal intensity on MPRAGE images (1.5 times the signal intensity compared with adjacent muscle). ${ }^{20}$ The volume of each plaque component, including calcification, LRNC, and IPH, and the corresponding percentage volume (volume of each carotid plaque component divided by plaque volume) were calculated. The volume of carotid plaque was calculated as the sum of the plaque area within slices multiplied by slice thickness. The length of carotid IPH was defined as the 


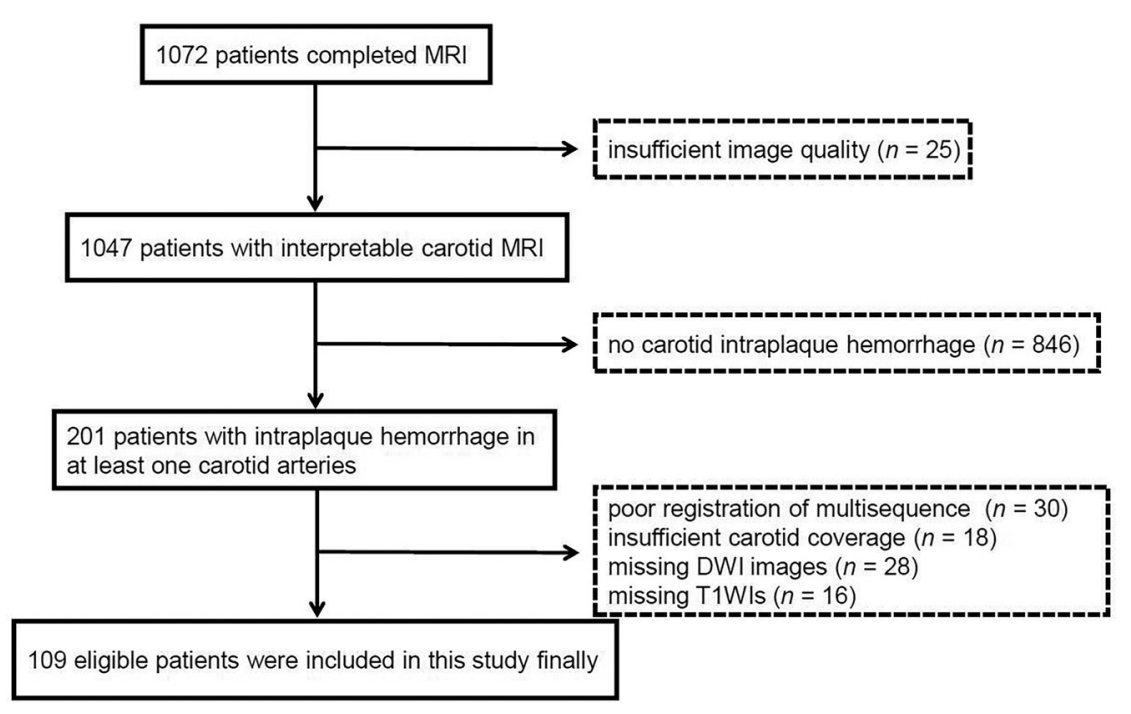

FIG 1. Flow chart of the study sample.

Table 2: Clinical characteristics of study population

\begin{tabular}{lccc} 
& \multicolumn{2}{c}{ Mean \pm SD or (No.) (\%) } & \\
\cline { 2 - 3 } & Patients with & Patients without & \\
& $\mathrm{ACI}(\boldsymbol{n}=\mathbf{4 0})$ & $\mathrm{ACl}(\boldsymbol{n}=69)$ & $\boldsymbol{P}$ Value $\mathbf{a}^{\mathrm{a}}$ \\
\hline Age (yr) & $64.2 \pm 10.2$ & $68.3 \pm 9.5$ & .035 \\
Sex, male & $37(92.5)$ & $59(85.5)$ & .366 \\
Body mass index $\left(\mathrm{kg} / \mathrm{m}^{2}\right)$ & $25.1 \pm 3.6$ & $24.0 \pm 2.7$ & .177 \\
History of smoking & $30(75.0)$ & $38(55.1)$ & .038 \\
History of hypertension & $33(82.5)$ & $58(84.1)$ & .833 \\
Systolic blood pressure $(\mathrm{mm} \mathrm{Hg})$ & $144.2 \pm 20.9$ & $143.9 \pm 21.3$ & .840 \\
Diastolic blood pressure $(\mathrm{mm} \mathrm{Hg})$ & $85.2 \pm 9.2$ & $85.8 \pm 14.1$ & .720 \\
History of hyperlipidemia & $21(52.5)$ & $39(56.5)$ & .684 \\
LDL (mmol/L) & $2.7 \pm 1.0$ & $3.0 \pm 1.0$ & .182 \\
HDL (mmol/L) & $1.1 \pm 0.2$ & $1.1 \pm 0.2$ & .780 \\
TC (mmol/L) & $4.3 \pm 1.2$ & $4.7 \pm 1.1$ & .161 \\
TG (mmol/L) & $1.9 \pm 1.2$ & $2.0 \pm 1.1$ & .519 \\
History of diabetes mellitus & $15(37.5)$ & $25(36.2)$ & .895 \\
History of coronary heart disease & $11(27.5)$ & $14(20.3)$ & .388 \\
Statin use & $15(37.5)$ & $25(36.2)$ & .895 \\
Antihypertension medication use & $28(70.0)$ & $49(71.0)$ & .911 \\
TIA & $12(30.0)$ & $32(46.4)$ & .108 \\
Recent stroke & $28(70.0)$ & $37(53.6)$ & .108 \\
\hline
\end{tabular}

Note:-LDL indicates low-density lipoprotein cholesterol; HDL, high-density lipoprotein cholesterol; TC, total cholesterol; TG, triglycerides; TIA, transient ischemia attack.

${ }^{\text {a }} P$ values were calculated by the Mann-Whitney $U$ test or Fisher exact test. subjects with total luminal occlusion. In analyzing these subjects, we usually reviewed only the slices without luminal occlusion. Brain MR images were evaluated by 2 experienced radiologists who had $>3$ years of experience in neurovascular imaging with consensus, blinded to clinical information and carotid MR images. The presence or absence of ACI lesions in the internal carotid artery territory was determined on the hemisphere ipsilateral to carotid IPH. ACI was defined as lesions that showed hyperintensity on DWI $\left(b=1000 \mathrm{~s} / \mathrm{mm}^{2}\right)$, but iso- or hypointensity on T1-weighted images.

\section{Statistical Analysis}

For patients with bilateral carotid IPHs, the MR imaging features of the carotid artery associated with symptoms were selected for final statistical analysis. The continuous variables were presented as mean $\pm \mathrm{SD}$ or median and interquartile range, and the binary variables were expressed as count and percentage. The plaque features on carotid MR imaging were compared between patients with and without ACI using the independent $t$ test, Mann-Whitney $U$ test, $\chi^{2}$ test, or Fisher exact test. Univariate and multivariate logistic regressions were performed to calculate the $\mathrm{OR}$ and corresponding 95\% CI of the carotid SIR of carotid IPH against muscle features on different sequences in discriminating the presence of ipsilateral ACI lesions before and after adjustment for confounding factors. $P<.05$ was considered statistically significant. All statistical analyses were performed using SPSS 16.0 (IBM, Armonk, New York). number of slices with carotid IPH on MPRAGE images multiplied by slice thickness. The ROIs were drawn to enclose the IPH region and the adjacent muscle, respectively, on each axial MPRAGE image with IPH and were automatically mapped to other MR imaging sequences (On-line Figure). The maximum signal intensity of carotid IPH and the mean signal intensity of the adjacent sternocleidomastoid muscle on T1-weighted, TOF, and MPRAGE images were recorded.

The SIR of carotid IPH against muscle on different imaging sequences was calculated by dividing the maximum signal intensity of the IPH by the mean signal intensity of the sternocleidomastoid muscle on each axial image. ${ }^{21}$ Luminal stenosis was measured by radiologists on TOF MR angiography using the NASCET criteria. ${ }^{22}$ In the present study, we included some

\section{RESULTS}

The flow chart of patient recruitment in this study is shown in Fig 1. In total, 201 patients with carotid IPH in at least one carotid artery were recruited in this study. Of the 201 patients, 92 patients were excluded due to the following reasons: 1) poor registration of multisequences $(n=30) ; 2)$ insufficient coverage for carotid images $(n=18) ; 3)$ missing DWI images $(n=28)$; and 4) missing carotid T1-weighted images $(n=16)$. Finally, 109 patients with carotid IPH were qualified for the final analysis.

\section{Clinical Characteristics}

Of the 109 patients, 40 (36.7\%) had ACI lesions in the ipsilateral hemispheres of carotid IPH, of which 28 (70\%) had a recent stroke and $12(30 \%)$ had a transient ischemic attack. The clinical 
characteristics between patients with and without ipsilateral ACI lesions are presented in Table 2. Patients with ipsilateral ACI lesions were younger (mean, $64.2 \pm 10.2$ versus $68.3 \pm 9.5$ years of age, $P=.035)$ and had a higher prevalence of smoking $(75.0 \%$ versus $55.1 \%, P=.038$ ) compared with those without. No significant differences were found in other clinical characteristics between patients with and without ipsilateral ACI.

\section{Plaque Morphology and Component Characteristics}

The comparison results on the carotid plaque morphological and compositional characteristics between patients with and without ipsilateral ACI lesions are shown in Table 3. Patients with ipsilateral ACI lesions had significantly greater mean wall area $(48.3 \pm$

Table 3: Comparison of carotid plaque morphologic and compositional characteristics between patients with and without ipsilateral ACI lesions

\begin{tabular}{|c|c|c|c|}
\hline & \multicolumn{2}{|c|}{$\begin{array}{c}\text { Mean } \pm \text { SD. (No.) (\%) or Median } \\
(25-75 \text { Percentiles) }\end{array}$} & \multirow[b]{2}{*}{$\begin{array}{c}P \\
\text { Value }\end{array}$} \\
\hline & $\begin{array}{l}\text { Patients with } \mathrm{ACl} \\
(n=40)\end{array}$ & $\begin{array}{l}\text { Patients without } \mathrm{ACl} \\
(n=69)\end{array}$ & \\
\hline Mean lumen area $\left(\mathrm{mm}^{2}\right)$ & $35.5 \pm 13.5$ & $36.5 \pm 12.4$ & .555 \\
\hline Mean wall area $\left(\mathrm{mm}^{2}\right)$ & $48.3 \pm 14.9$ & $41.5 \pm 11.1$ & .028 \\
\hline Mean total vessel area $\left(\mathrm{mm}^{2}\right)$ & $83.8 \pm 20.6$ & $78.0 \pm 18.6$ & .172 \\
\hline Mean wall thickness (mm) & $1.9 \pm 0.6$ & $1.6 \pm 0.4$ & .010 \\
\hline Mean normalized wall index (\%) & $57.9 \pm 11.3$ & $53.6 \pm 9.1$ & .087 \\
\hline Total luminal occlusion & $21(52.5 \%)$ & $11(15.9 \%)$ & $<.001$ \\
\hline Plaque volume $\left(\mathrm{mm}^{2}\right)$ & $1240.7 \pm 535.2$ & $1064.4 \pm 414.1$ & .159 \\
\hline Presence of calcification & $33(82.5)$ & $60(87.0)$ & .526 \\
\hline Presence of FCR & $14(35.0)$ & $23(33.3)$ & .859 \\
\hline Volume of calcification $\left(\mathrm{mm}^{3}\right)^{\mathrm{b}}$ & $35.8(12.4-87.3)$ & $36.2(15.8-79.6)$ & .794 \\
\hline Percentage calcification volume (\%) ${ }^{\mathrm{b}}$ & $3.1(1.3-6.4)$ & $3.5(1.9-7.7)$ & .312 \\
\hline Volume of LRNC $\left(\mathrm{mm}^{3}\right)^{\mathrm{b}}$ & 312.7 (174.1-625.3) & $227.7(122.1-351.4)$ & .038 \\
\hline Percentage LRNC volume (\%) ${ }^{\mathrm{b}}$ & $29.4(17.9-42.1)$ & $23.6(14.0-34.0)$ & .039 \\
\hline Volume of IPH $\left(\mathrm{mm}^{3}\right)^{\mathrm{b}}$ & $93.2(31.4-201.2)$ & $56.4(21.9-104.9)$ & .016 \\
\hline Percentage IPH volume (\%) ${ }^{\mathrm{b}}$ & $7.9(3.0-17.0)$ & $5.4(2.5-10.1)$ & .074 \\
\hline IPH length (mm) & $12.0(4.5-17.5)$ & $10.0(4.0-14.0)$ & .101 \\
\hline
\end{tabular}

Note:-FCR indicates fibrous cap rupture.

${ }^{a} P$ values are based on the Mann-Whitney $U$ test or Fisher exact test between patients with and without ipsilateral $\mathrm{ACl}$ lesions.

${ }^{\mathrm{b}}$ Only patients with the corresponding component present were included in the comparison.
14.9 versus $\left.41.5 \pm 11.1 \mathrm{~mm}^{2}, P=.028\right)$, mean wall thickness $(1.9 \pm 0.6$ versus $1.6 \pm 0.4 \mathrm{~mm}, P=.010)$, and a higher prevalence of total luminal occlusion of the carotid artery $(52.5 \%$ versus $15.9 \%, P<.001)$ than those without. There were no statistically significant differences in other carotid plaque burden measurements, including lumen area, total vessel area, mean normalized wall index and carotid plaque volume between the two groups (all $P>.05$ ). Of all 109 patients, 93 (85.3\%) had carotid calcification and 37 (33.9\%) had carotid fibrous cap rupture. There were no significant differences in the prevalence of calcification $(P=.526)$ and fibrous cap rupture $(P=.859)$ between patients with and without ipsilateral ACI lesions. Patients with ipsilateral ACI lesions had a significantly larger volume of LRNC (312.7 [174.1-625.3] versus 227.7 [122.1-351.4] $\left.\mathrm{mm}^{3}, P=.038\right)$, a larger percentage LRNC volume (29.4\% versus $23.6 \%, P=.039)$, and a larger IPH volume (93.2 [31.4201.2] versus 56.4 [21.9-104.9] $\mathrm{mm}^{3}$, $P=.016)$ than those without. No significant differences were found in the percentage of IPH volume, volume of calcification, percentage of calcification volume, and carotid plaque length between the two groups (all, $P>.05$ ).

\section{IPH Signal Characteristics}

Patients with ipsilateral ACI lesions were found to have significantly higher SIRs of carotid IPH (1.44 [1.14-1.82] versus 1.27 [1.06-1.55], $P=.022$ ) on T1-weighted images than those without (Fig 2). No significant differences were found in the SIRs of carotid IPH on TOF (1.36 [1.11-1.72] versus 1.19 [0.92-1.58], $P=.096)$ and

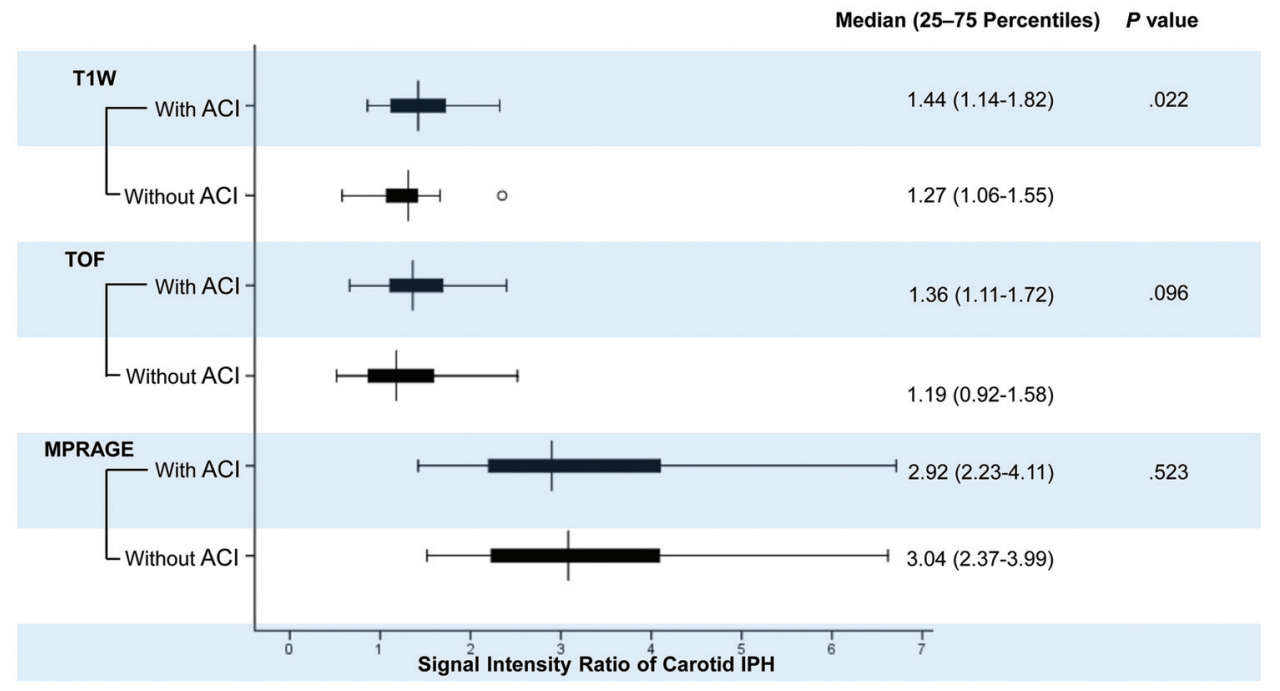

FIG 2. Boxplots of the SIR of carotid IPH on different T7-weighted MR imaging sequences in patients with and without ACl. Patients with ipsilateral ACI lesions had significantly higher SIRs of carotid IPH on TI-weighted images than those without. 
Table 4: Association between signal intensity ratio of carotid IPH and the presence of ipsilateral ACI lesions

\begin{tabular}{|c|c|c|c|c|c|c|c|c|c|c|c|c|}
\hline \multirow{3}{*}{$\begin{array}{l}\text { SIR }_{\text {IPH-to-muscle }} \text { on } \\
\text { MR Image }\end{array}$} & \multicolumn{12}{|c|}{ Presence of $\mathrm{ACl}$} \\
\hline & \multicolumn{3}{|c|}{ Univariate Regression } & \multicolumn{3}{|c|}{ Multivariate Model $1^{\mathrm{a}}$} & \multicolumn{3}{|c|}{ Multivariate Model $2^{a}$} & \multicolumn{3}{|c|}{ Multivariate Model $3^{a}$} \\
\hline & OR & $95 \% \mathrm{Cl}$ & $P^{\mathrm{b}}$ & OR & $95 \% \mathrm{Cl}$ & $P^{\mathrm{b}}$ & OR & $95 \% \mathrm{Cl}$ & $P^{\mathrm{b}}$ & OR & $95 \% \mathrm{Cl}$ & $P^{\mathrm{b}}$ \\
\hline T1-weighted & 4.08 & $1.34-12.40$ & .013 & 3.34 & $1.08-10.31$ & .036 & 3.12 & $0.96-10.11$ & .058 & 1.57 & $0.48-5.20$ & .458 \\
\hline TOF & 1.98 & $0.80-4.93$ & .141 & 2.54 & $0.94-6.82$ & .065 & 2.70 & $0.90-8.13$ & .077 & 1.21 & $0.37-3.93$ & .756 \\
\hline MPRAGE & 0.86 & $0.51-1.45$ & .561 & 0.85 & $0.48-1.48$ & .557 & 0.98 & $0.53-1.79$ & .941 & 0.56 & $0.28-1.12$ & .101 \\
\hline
\end{tabular}

Note:-SIR 1 PH-to-muscle indicates the maximum signal intensity of carotid IPH/the mean signal intensity of the sternocleidomastoid muscle.

${ }^{a}$ Multivariate model 1 was adjusted for age, sex, and history of smoking; model 2 was further adjusted using model 1 and total luminal occlusion of the carotid artery. Model 3 was further adjusted using model 1 and the volume of carotid IPH and LRNC.

${ }^{\mathrm{b}}$ Associations between patients with and without ipsilateral $\mathrm{ACl}$ lesions were assessed using the OR values.

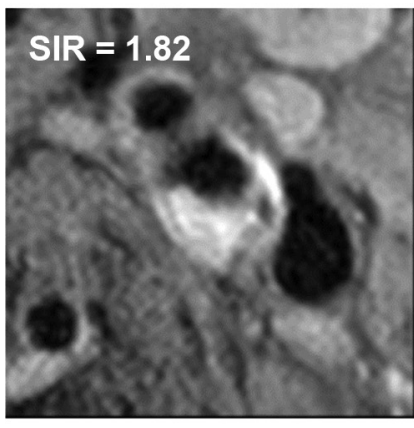

T1W

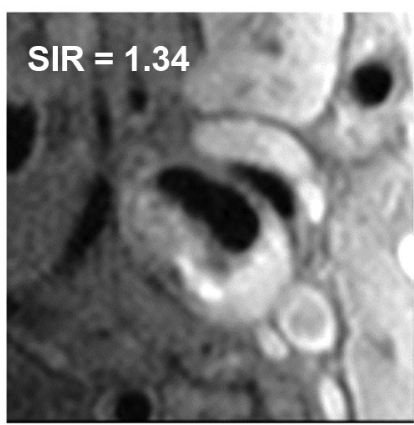

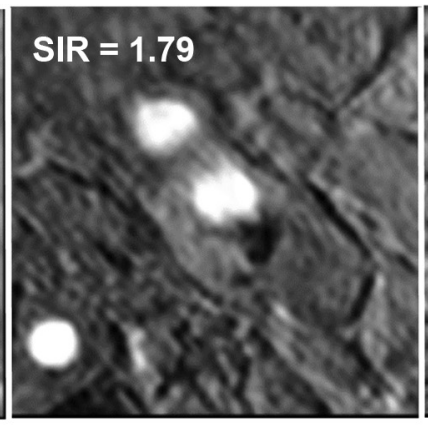

TOF

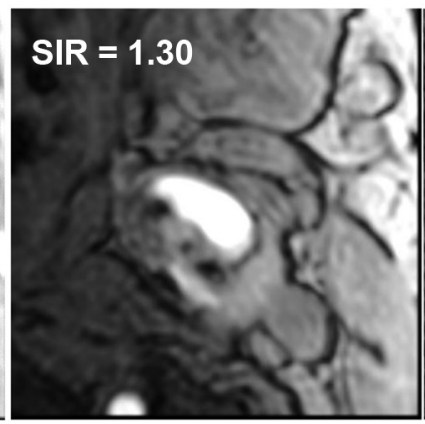

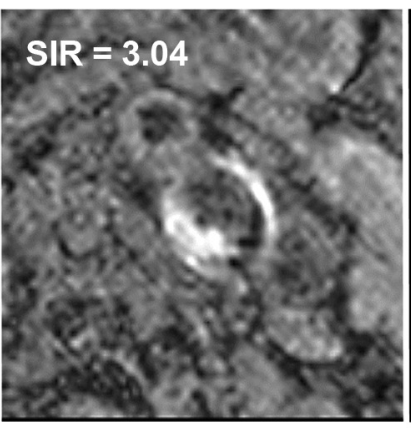

MPRAGE

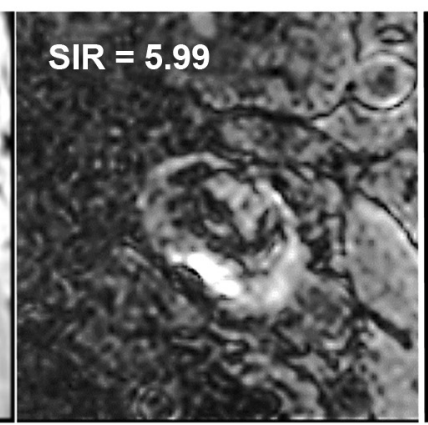

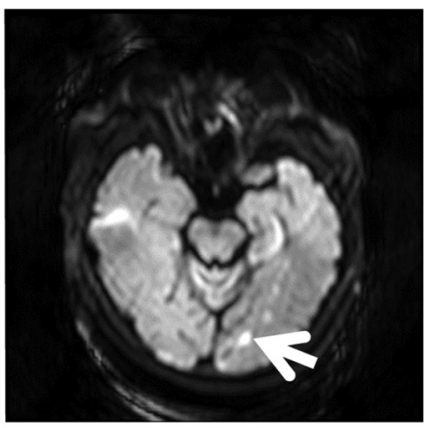

DWI

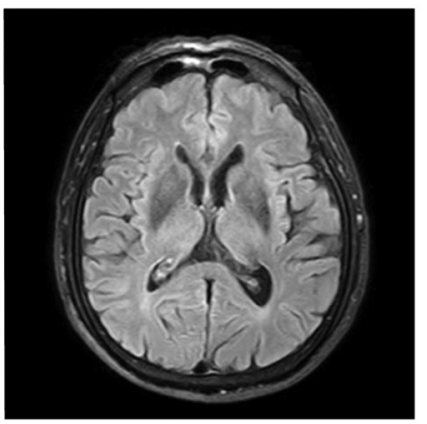

FIG 3. Comparison of the SIR of carotid IPH on Tl-weighted MR imaging sequences, including T1-weighted, TOF, and MPRAGE, between patients with $\mathrm{ACl}$ ( 4 images above, hyperintense on DWI as the arrow indicates) and without ACI ( 4 images below, no abnormality on DWI). It shows that the patient with the higher SIR on TI-weighted images had ACI lesions in the ipsilateral hemisphere.

MPRAGE (2.92 [2.23-4.11] versus 3.04 [2.37-3.99], $P=.523)$ images between patients with and without ipsilateral ACI lesions (Fig 2).

\section{Association between SIRs of Carotid IPH and the Ipsilateral ACI}

Univariate regression analysis (Table 4) revealed that the SIRs of carotid IPH on T1-weighted images were significantly associated with the presence of ipsilateral ACI lesions (OR, 4.08; 95\% CI, 1.34-12.40; $P=.013$ ). After adjustment for clinical confounding factors of age, sex, and history of smoking (model 1), this association remained statistically significant (OR, 3.34; 95\% CI, 1.08$10.31 ; P=.036$ ). After further adjustment for total luminal occlusion of the carotid artery (model 2), this association appeared marginally significant (OR, 3.12; 95\% CI, 0.96-10.11; $P=.058)$. However, after further adjustment for the volume of carotid IPH and LRNC (model 3), this association was not significant (OR, 1.57; 95\% CI, 0.48-5.20; $P=.458$ ). Figure 3 shows that a patient with higher SIRs of carotid IPH against muscle on T1-weighted images had ACI lesions in the ipsilateral hemisphere on DWI. There were no significant associations between SIRs of carotid IPH on TOF and MPRAGE images and the presence of ACI lesions before and after adjustment for clinical confounding factors, total luminal occlusion of the carotid artery, and the size of carotid IPH and LRNC (all, $P>.05$; Table 4).

\section{DISCUSSION}

The present study investigated the association between the SIRs of carotid IPH on different T1-weighted MR imaging sequences and ipsilateral ACI lesions in patients with carotid hemorrhagic plaques using multicontrast MR vessel wall imaging. Although we found that patients with ipsilateral ACI lesions had significantly higher SIRs of carotid IPH on T1-weighted images than those without statistically, there was still an overlap in the SIRs of carotid IPH on T1-weighted images between the 
two groups. Only extremely low (range, 1.06-1.14) or high (range, 1.55-1.82) SIR values will allow a fairly accurate prediction of whether an ACI is present. Further studies are warranted combining SIRs of IPH with other metrics for predicting ACI, particularly for those IPHs with SIRs within this overlap. Furthermore, there was a significant association between the SIR of carotid IPH on T1-weighted images and the presence of ipsilateral ACI lesions, which was independent of traditional risk factors. After further adjustment for total luminal occlusion of the carotid artery, this association appeared marginally significant. This finding implies that the association between the SIRs of IPH on T1-weighted images and ACI might be influenced by the luminal occlusion. However, this association was not significant after further adjustment for the volume of carotid IPH and LRNC, suggesting that the differences of carotid SIRs on T1-weighted images between the two groups might be, in part, attributed to the different sizes of carotid IPH and LRNC. Our findings might provide additional imaging information in predicting the presence of cerebrovascular ischemic events beyond the mere presence of IPH.

In the present study, patients with ACI lesions were found to be younger than those without, which was consistent with the study by Turin et $\mathrm{al}^{23}$ in which a lifetime risk of stroke was higher in younger age than in older age. Another recent study by Feigin et $\mathrm{al}^{24}$ indicated that the global lifetime risk of stroke declined with age in patients older than 70 years, due to agerelated competing risks from other diseases. ${ }^{25}$ In addition, the present study also demonstrated that patients with ipsilateral ACI lesions were more likely to smoke than those without, which was in line with previous studies. Wolf et $\mathrm{al}^{26}$ and Donnan et $\mathrm{al}^{27}$ have reported that cigarette smoking was an independent risk factor for cerebral infarct. Nicotine has been considered a key component that contributes to the influence of cigarette smoking on the cerebral infarct. Li et $\mathrm{al}^{28}$ demonstrated that mitochondrial oxidative stress induced by nicotine may have important implications for the pathogenesis and pathophysiology of ischemic stroke.

In this study, we assessed the association between the SIRs of carotid IPH on different MR images and ipsilateral ACI lesions in symptomatic patients with carotid hemorrhagic plaques. We found that the SIRs of carotid IPH on T1-weighted images were associated with the presence of ipsilateral ACI lesions in symptomatic patients regardless of clinical confounding factors, including age, sex, and a history of smoking. However, this association was not significant after further adjustment for total luminal occlusion of the carotid artery and the volume of carotid IPH and LRNC. In contrast, previous studies that included patients both with and without carotid IPH also demonstrated the association between the T1-weighted signal of carotid plaque and cerebral ischemic lesions. Takemoto et $\mathrm{al}^{29}$ reported that a higher T1weighted signal intensity of carotid plaque was present more often in symptomatic patients with lesions positive for cerebral ischemia on DWI. In a study of patients with carotid artery stenosis scheduled for carotid endarterectomy or carotid artery stenting, Kurosaki et $\mathrm{al}^{30}$ documented that plaques with higher signal intensity on T1-weighted images were at higher risk of causing ischemic events. However, the mechanism for the association between the SIRs of carotid IPH on T1-weighted images and the presence of ACI remained unclear.

Previous studies have demonstrated that the signal intensity of IPH was associated with the age of hemorrhage within plaques, and high signal intensity of IPH on T1-weighted images may indicate a recent hemorrhage. ${ }^{31}$ In addition, the increasing size of IPH, which means a higher concentration of methemoglobin locally within the plaque, may also play a role in the occurrence of stronger SIRs of IPH on T1-weighted images. A study by Leung and Moody ${ }^{32}$ revealed that erythrocyte degradation caused intraplaque deposition of methemoglobin, which had a strong T1-shortening effect. In the present study, no significant association was found between SIRs of carotid IPH on T1-weighted images and the presence of ACI when adjusted for clinical confounding factors and carotid IPH and LRNC volume, suggesting that the difference in carotid SIRs on T1-weighted images between the two patient groups might be, in part, attributed to the different sizes of IPH and LRNC.

In addition, we found that patients with ACI lesions had significantly larger carotid IPH and LRNC, respectively, than those without. Takaya et $\mathrm{al}^{5}$ reported that carotid plaques with a larger area of IPH (hazard ratio for a $10-\mathrm{mm}^{2}$ increase, 2.6; 95\% CI, 1.4-4.6; $P=.006$ ) were significantly associated with subsequent ipsilateral carotid cerebrovascular events. However, the mechanism for this phenomenon remained uncertain. Previous studies indicated that the volume of IPH was significantly independently associated with minor fibrous cap disruption in carotid arteries (OR, 2.152; 95\% CI, 1.241-3.730; $P=.006) .{ }^{33}$ Another study by Zhao et $\mathrm{al}^{34}$ indicated that patients with larger LRNC plaques might have larger cerebral infarcts. This phenomenon might be because that the large LRNC volume would accompany a larger fibrous cap area and increase the risk of rupture. Therefore, the size of carotid IPH and LRNC may influence, in part, the association between SIRs of carotid IPH and ACI. In this study, it is possible that carotid SIRs on T1-weighted imaging may combine both IPH and LRNC. Histologically, it is hypothesized that IPH occurs within the large lipid-rich necrotic core. Although the IPH was outlined on MPRAGE images, it is possible to include the LRNC in the ROI of IPH when the IPH is inhomogeneous. Our findings suggest that the SIR of IPH might be a surrogate for the size of the IPH or LRNC in predicting ACI.

An interesting issue we observed is that the SIRs of carotid IPH on MPRAGE images in patients with ipsilateral ACI lesions tended to be similar to those without (Median, 2.92; 25-75 Percentiles, 2.23-4.11 versus Median, 3.04; 25-75 Percentiles, 2.37-3.99, $P=.523$ ), a finding that was inconsistent with those in previous studies. A recent study by Wang et $\mathrm{al}^{16}$ indicated that carotid IPH signals on MPRAGE images on the symptomatic side were significantly higher (mean, $5.8 \pm 2.4$ versus $4.7 \pm 1.8, P=.004)$ than those on the asymptomatic side in symptomatic patients with bilateral carotid IPHs. There were a number of methodologic differences, including different sampling techniques and sample sizes, between the study by Wang et $\mathrm{al}^{16}$ and the present study. For example, in our study, patients included were divided into two groups due to the presence or absence of ACI lesions on DWI, while in the study by Wang et 
al, carotid plaques with IPH were divided into symptomatic and asymptomatic sides according to the recent symptoms, and the ACI lesions in that study on the symptomatic side were not distinct. More studies need to be performed to investigate the differences in this issue.

There are some limitations to this study. First, it only investigated the association between the SIRs of carotid IPH and ischemic stroke but did not analyze the difference in the age of IPH between patients with and without ACI lesions. The age of IPH may influence the association between the SIRs of IPH and ipsilateral ACI lesions. Future studies are warranted. Second, the current in-plane spatial resolution used in this study was $0.55 \mathrm{~mm}$, which is limited to accurately quantifying small IPHs. Third, in this study, approximately one-fourth of the 201 patients were excluded due to "poor registration of multisequences," suggesting that the method, at least in a certain number of subjects, is just not feasible. Future studies with larger samples and more improved technical methods are needed. Finally, although patients were recruited from a multicenter study, carotid IPH signal measurements on different sequences were performed on a single platform using a unified MR imaging protocol. Future studies using similar but not identical techniques are needed.

\section{CONCLUSIONS}

The signal intensity ratio of carotid IPH on T1-weighted images is associated with ACI in symptomatic patients with carotid hemorrhagic plaques. This association is independent of traditional risk factors but not of the size of plaque compositions. The possibility of applying T1 signals of carotid IPH to predict subsequent cerebrovascular ischemic events needs to be prospectively verified.

Disclosures: Chun Yuan-RELATED: Grant: Philips Healthcare; UNRELATED: Grants/Grants Pending: National Institutes of Health, Philips Healthcare.* *Money paid to the institution.

\section{REFERENCES}

1. Liu L, Wang D, Wong KS, et al. Stroke and stroke care in China: huge burden, significant workload, and a national priority. Stroke 2011;42:3651-54 CrossRef Medline

2. Barnett HJ, Gunton RW, Eliasziw M, et al. Causes and severity of ischemic stroke in patients with internal carotid artery stenosis. JAMA 2000;283:1429-36 CrossRef Medline

3. Underhill HR, Hatsukami TS, Cai J, et al. A noninvasive imaging approach to assess plaque severity: the carotid atherosclerosis score. AJNR Am J Neuroradiol 2010;31:1068-75 CrossRef Medline

4. Hosseini AA, Kandiyil N, Macsweeney ST, et al. Carotid plaque hemorrhage on magnetic resonance imaging strongly predicts recurrent ischemia and stroke. Ann Neurol 2013;73:774-84 CrossRef Medline

5. Takaya N, Yuan C, Chu B, et al. Association between carotid plaque characteristics and subsequent ischemic cerebrovascular events: a prospective assessment with MRI-initial results. Stroke 2006;37: 818-23 CrossRef Medline

6. Singh N, Moody AR, Gladstone DJ, et al. Moderate carotid artery stenosis: MR imaging-depicted intraplaque hemorrhage predicts risk of cerebrovascular ischemic events in asymptomatic men. Radiology 2009;252:502-08 CrossRef Medline

7. McNally JS, McLaughlin MS, Hinckley PJ, et al. Intraluminal thrombus, intraplaque hemorrhage, plaque thickness, and current smoking optimally predict carotid stroke. Stroke 2015;46:84-90 CrossRef Medline
8. Fryer JA, Myers PC, Appleberg M. Carotid intraplaque hemorrhage: significance of neovascularity. J Vasc Surg 1987;6:341-49 CrossRef Medline

9. Dong L, Underhill HR, Yu W, et al. Geometric and compositional appearance of atheroma in an angiographically normal carotid artery in patients with atherosclerosis. AJNR Am J Neuroradiol 2010;31:311-16 CrossRef Medline

10. Cai Y, He L, Yuan C, et al. Atherosclerotic plaque features and distribution in bilateral carotid arteries of asymptomatic elderly population: a 3D multicontrast MR vessel wall imaging study. Eur J Radiol 2017;96:6-11 CrossRef Medline

11. Sun J, Underhill HR, Hippe DS, et al. Sustained acceleration in carotid atherosclerotic plaque progression with intraplaque hemorrhage: a long-term time course study. JACC Cardiovasc Imaging 2012;5:798-804 CrossRef Medline

12. Takaya N, Yuan C, Chu B, et al. Presence of intraplaque hemorrhage stimulates progression of carotid atherosclerotic plaques: a high-resolution magnetic resonance imaging study. Circulation 2005;111:2768-75 CrossRef Medline

13. Moody AR, Murphy RE, Morgan PS, et al. Characterization of complicated carotid plaque with magnetic resonance direct thrombus imaging in patients with cerebral ischemia. Circulation 2003;107: 3047-52 CrossRef Medline

14. Yamada K, Yoshimura S, Shirakawa M, et al. Asymptomatic moderate carotid artery stenosis with intraplaque hemorrhage: progression of degree of stenosis and new ischemic stroke. J Clin Neurosci 2019;63:95-99 CrossRef Medline

15. Yamada K, Kawasaki M, Yoshimura S, et al. High-intensity signal in carotid plaque on routine 3D-TOF-MRA is a risk factor of ischemic stroke. Cerebrovasc Dis 2016;41:13-18 CrossRef Medline

16. Wang X, Sun J, Zhao X, et al; CARE-II study investigators. Ipsilateral plaques display higher $\mathrm{T} 1$ signals than contralateral plaques in recently symptomatic patients with bilateral carotid intraplaque hemorrhage. Atherosclerosis 2017;257:78-85 CrossRef Medline

17. Zhao X, Li R, Hippe DS, et al; CARE-II Investigators. Chinese Atherosclerosis Risk Evaluation (CARE II) study: a novel crosssectional, multicentre study of the prevalence of high-risk atherosclerotic carotid plaque in Chinese patients with ischaemic cerebrovascular events-design and rationale. Stroke Vasc Neurol 2017;2: 15-20 CrossRef Medline

18. Kerwin $\mathrm{W}, \mathrm{Xu} \mathrm{D}$, Liu F, et al. Magnetic resonance imaging of carotid atherosclerosis: plaque analysis. Top Magn Reson Imaging 2007;18:371-78 CrossRef Medline

19. Cai JM, Hatsukami TS, Ferguson MS, et al. Classification of human carotid atherosclerotic lesions with in vivo multicontrast magnetic resonance imaging. Circulation 2002;106:1368-73 CrossRef Medline

20. Li D, Zhao H, Chen $\mathrm{X}$, et al. Identification of intraplaque haemorrhage in carotid artery by simultaneous non-contrast angiography and intraPlaque haemorrhage (SNAP) imaging: a magnetic resonance vessel wall imaging study. Eur Radiol 2018;28:1681-86 CrossRef Medline

21. Eto A, Kinoshita Y, Matsumoto Y, et al. Relationship between the carotid plaque $\mathrm{T} 1$ relaxation time and the plaque-to-muscle signal intensity ratio on black-blood magnetic resonance imaging scans. $J$ Stroke Cerebrovasc Dis 2016;25:2580-84 CrossRef Medline

22. North American Symptomatic Carotid Endarterectomy Trial: methods, patient characteristics, and progress. Stroke 1991;22: 711-20 CrossRef Medline

23. Turin TC, Okamura T, Afzal AR, et al. Hypertension and lifetime risk of stroke. J Hypertens 2016;34:116-22 CrossRef Medline

24. Feigin VL, Nguyen G, Cercy K, et al; GBD 2016 Lifetime Risk of Stroke Collaborators. Global, regional, and country-specific lifetime risks of stroke. $N$ Engl J Med 2018;379:2429-37 CrossRef Medline

25. Naghavi M, Abajobir AA, Abbafati C, et al. Global, regional, and national age-sex specific mortality for 264 causes of death, 19802016: a systematic analysis for the Global Burden of Disease Study. Lancet 2017;390:1151-16 CrossRef 
26. Wolf PA, D'Agostino RB, Kannel WB, et al. Cigarette smoking as a risk factor for stroke: the Framingham Study. JAMA 1988;259:102529 Medline

27. Donnan GA, McNeil JJ, Adena MA, et al. Smoking as a risk factor for cerebral ischaemia. Lancet 1989;2:643-47 CrossRef Medline

28. Li C, Sun H, Arrick DM, et al. Chronic nicotine exposure exacerbates transient focal cerebral ischemia-induced brain injury. $J \mathrm{Appl}$ Physiol 2016;120:328-33 CrossRef Medline

29. Takemoto K, Ueba T, Takano K, et al. Quantitative evaluation using the plaque/muscle ratio index panels predicts plaque type and risk of embolism in patients undergoing carotid artery stenting. Clin Neurol Neurosurg 2013;115:1298-1303 CrossRef Medline

30. Kurosaki Y, Yoshida K, Fukumitsu R, et al. Carotid artery plaque assessment using quantitative expansive remodeling evaluation and
MRI plaque signal intensity. J Neurosurg 2016;124:736-42 CrossRef Medline

31. Chu B, Kampschulte A, Ferguson MS, et al. Hemorrhage in the atherosclerotic carotid plaque: a high-resolution MRI study. Stroke 2004;35:1079-84 CrossRef Medline

32. Leung G, Moody AR. MR imaging depicts oxidative stress induced by methemoglobin. Radiology 2010;257:470-76 CrossRef Medline

33. Cui Y, Qiao H, Ma L, et al. Association of age and size of carotid artery intraplaque hemorrhage and minor fibrous cap disruption: a high-resolution magnetic resonance imaging study. J Atheroscler Thromb 2018;25:1222-30 CrossRef Medline

34. Zhao H, Zhao X, Liu X, et al. Association of carotid atherosclerotic plaque features with acute ischemic stroke: a magnetic resonance imaging study. Eur J Radiol 2013;82:e465-70 CrossRef Medline 